\title{
Competitive Interactions of Two Ion Paired Salts with a Neutral Host to Form Two Non-Ion Paired Complexes
}

\author{
Feihe Huang, ${ }^{*,+, \ddagger}$ Jason W. Jones, ${ }^{\ddagger, \Phi}$ and Harry W. Gibson, ${ }^{, \neq}$ \\ ${ }^{\dagger}$ Department of Chemistry, Zhejiang University, Hangzhou 310027, P. R. China \\ ${ }^{\ddagger}$ Department of Chemistry, Virginia Polytechnic Institute \& State University, \\ Blacksburg, Virginia, 24061
}

\section{Supplemental Material (2 pages)}

The following derivation for complexation of paraquats (or other divalent cationic guests) with two univalent counterions in relatively low polarity organic solvents is based on the assumptions that a) the electrolyte exists in solution as a monomer in equilibrium with its ionized species $\mathrm{G}^{++} \mathrm{X}^{-}$and $\mathrm{G}^{++}$, b) it is the free doubly charged cation $\mathrm{G}^{++}$that forms the pseudorotaxane, c) the complex is completely dissociated, not ion paired and d) there are no other species present.

The treatment below takes into account the formation of free counterions, $\mathrm{X}^{-}$, and as a result their effect on complex formation.

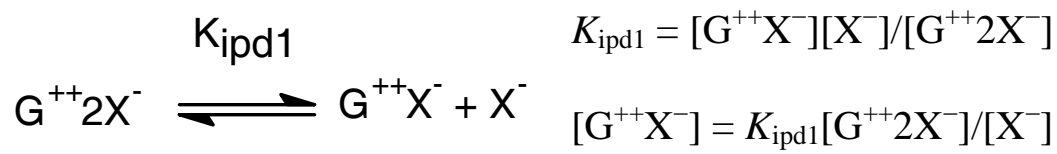

$$
\begin{aligned}
& \begin{array}{ll}
\mathrm{G}^{++} \mathrm{X}^{-} \stackrel{\mathrm{K}_{\mathrm{ipd} 2}}{\rightleftharpoons} \mathrm{G}^{++}+\mathrm{X}^{-} & K_{\mathrm{ipd} 2}=\left[\mathrm{G}^{++}\right]\left[\mathrm{X}^{-}\right] /\left[\mathrm{G}^{++} \mathrm{X}^{-}\right] \\
K_{\mathrm{ipdo}}=\left[\mathrm{G}^{++}\right]\left[\mathrm{X}^{-}\right]^{2} /\left[\mathrm{G}^{++} 2 \mathrm{X}^{-}\right]=K_{\mathrm{ipd} 1} K_{\mathrm{ipd} 2}
\end{array} \\
& \mathrm{G}^{++} 2 \mathrm{X}^{-} \stackrel{\mathrm{K}_{\text {ipdo }}}{\rightleftharpoons} \mathrm{G}^{++}+2 \mathrm{X}^{-} \quad\left[\mathrm{G}^{++}\right]=K_{\mathrm{ipdo}}\left[\mathrm{G}^{++} 2 \mathrm{X}^{-}\right] /\left[\mathrm{X}^{-}\right]^{2} \\
& \mathrm{H}+\mathrm{G}^{++} \stackrel{\mathrm{Kap}}{\rightleftharpoons} \mathrm{HG}^{++} \quad\left[\mathrm{HG}^{++}\right]=K_{\mathrm{ap}}[\mathrm{H}]\left[\mathrm{G}^{++}\right] \\
& K_{\mathrm{ap}}=\left[\mathrm{HG}^{++}\right]\left[\mathrm{X}^{-}\right]^{2} / K_{\mathrm{ipdo}}\left[\mathrm{G}^{++} 2 \mathrm{X}^{-}\right][\mathrm{H}]
\end{aligned}
$$

$$
\begin{aligned}
& K_{\text {ipd1 }} / K_{\text {ipdo }}=\left[\mathrm{G}^{++} \mathrm{X}^{-}\right] /\left[\mathrm{G}^{++}\right]\left[\mathrm{X}^{-}\right] \\
& \begin{aligned}
{\left[\mathrm{X}^{-}\right]=} & 2\left(\left[\mathrm{HG}^{++}\right]+\left[\mathrm{G}^{++}\right]\right)+\left[\mathrm{G}^{++} \mathrm{X}^{-}\right] \\
& =2\left\{K_{\mathrm{ap}}\left[\mathrm{G}^{++}\right][\mathrm{H}]+K_{\mathrm{ipdo}}\left[\mathrm{G}^{++} 2 \mathrm{X}^{-}\right] /\left[\mathrm{X}^{-}\right]^{2}\right\}+K_{\mathrm{ipd} 1}\left[\mathrm{G}^{++} 2 \mathrm{X}^{-}\right] /\left[\mathrm{X}^{-}\right] \\
& =2\left\{K_{\mathrm{ap}} K_{\text {ipdo }}\left[\mathrm{G}^{++} 2 \mathrm{X}^{-}\right][\mathrm{H}] /\left[\mathrm{X}^{-}\right]^{2}+K_{\mathrm{ipdo}}\left[\mathrm{G}^{++} 2 \mathrm{X}^{-}\right] /\left[\mathrm{X}^{-}\right]^{2}\right\}+K_{\mathrm{ipd} 1}\left[\mathrm{G}^{++} 2 \mathrm{X}^{-}\right] /\left[\mathrm{X}^{-}\right]
\end{aligned}
\end{aligned}
$$




$$
=\left\{\left[\mathrm{G}^{++} 2 \mathrm{X}^{-}\right] /\left[\mathrm{X}^{-}\right]^{2}\right\}\left\{2 K_{\mathrm{ipdo}}\left(K_{\mathrm{ap}}[\mathrm{H}]+1\right)+K_{\mathrm{ipd} 1}\left[\mathrm{X}^{-}\right]\right\}
$$

If $2 K_{\text {ipdo }}\left(1+K_{\mathrm{ap}}[\mathrm{H}]\right)>>K_{\mathrm{ipd} 1}\left[\mathrm{X}^{-}\right]$, Eq.1a is reduced to Eq. 1 b, which is identical to Eq. 1c in the JACS paper ${ }^{\mathrm{S} 1}$ and the data can be treated in the same manner as outlined there, i. e., by use of Eq. 1c (Eq. 1d in that paper). Eq. 2 of the present text corresponds to the same derivation.

$$
\begin{aligned}
& {\left[\mathrm{X}^{-}\right]=\left\{\left[\mathrm{G}^{++} 2 \mathrm{X}^{-}\right]\left[2 K_{\text {ipdo }}\left(K_{\mathrm{ap}}[\mathrm{H}]+1\right)\right\}^{1 / 3}\right.} \\
& {\left[\mathrm{HG}^{++}\right] /\left[\mathrm{G}^{++} 2 \mathrm{X}^{-}\right]^{1 / 3}=K_{\mathrm{ap}} K_{\mathrm{ipd}}^{1 / 3}[\mathrm{H}] /\left\{2\left(1+K_{\mathrm{ap}}[\mathrm{H}]\right)\right\}^{2 / 3}}
\end{aligned}
$$

If $K_{\mathrm{ap}}=100 \mathrm{M}^{-1}$ and $[\mathrm{H}]=0.50 \mathrm{mM}$, then $2\left(1+K_{\mathrm{ap}}[\mathrm{H}]\right)=2.1$. Since the maximum $\left[\mathrm{G}^{++} 2 \mathrm{X}^{-}\right]_{0}$ used here is $1.00 \mathrm{mM}$, then $\left[\mathrm{X}^{-}\right]<2.00 \mathrm{mM}$. Thus, at this extreme of the concentration range, to satisfy the inequality noted above,

$$
2.1 K_{\text {ipdo }}>>\left(2 \times 10^{-3}\right) K_{\text {ipd } 1} \text {. }
$$

Or equivalently $K_{\mathrm{ipdo}} / K_{\mathrm{ipd} 1}=K_{\mathrm{ipd} 1} K_{\mathrm{ipd} 2} / K_{\mathrm{ipd} 1}=K_{\mathrm{ipd} 2}>>1 \times 10^{-3}$.

Higher values of [H] enable even lower values of $K_{\mathrm{ipd} 2}$ to still lead to Eq. 1c; e.g., at $[\mathrm{H}]=5.0 \mathrm{mM}$, to simplify Eq. 1 a to Eq. $1 \mathrm{~b} K_{\mathrm{ipd} 2}>>6.7 \times 10^{-4} \mathrm{M}^{-1}$.

Generally in our earlier work, ${ }^{\mathrm{S} 1}$ as $\left[\mathrm{G}^{++} 2 \mathrm{X}^{-}\right]_{0}$ increased so also did $[\mathrm{H}]_{\mathrm{o}}$. The maximum $\left[\mathrm{G}^{++} 2 \mathrm{X}^{-}\right]_{0}$ was $10.0 \mathrm{mM}$, while the maximum $[\mathrm{H}]_{0}$ was $30.0 \mathrm{mM}$. $[\mathrm{H}]_{\mathrm{uc}}$ ranged from 0.368 to $23.7 \mathrm{mM}$, while total uncomplexed guest concentration ranged from 0.240 to $5.17 \mathrm{mM}$.

The good fit previously found using Eq. 1d in reference S1 supports the validity of this approximation under these conditions.

\section{REFERENCE}

1. Huang, F.; Jones, J. W.; Slebodnick, C.; Gibson, H. W. J. Am. Chem. Soc. 2003, 125, 14458-14464. 